\title{
A Novel Feature Extraction Method for Soft Faults in Nonlinear Analog Circuits Based on LMD-GFD and KPCA
}

\author{
Xinmiao LU*, Jiaxu WANG, Qiong WU, Yuhan WEI, Yanwen SU
}

\begin{abstract}
To obtain feature information of soft faults in non-linear analog circuits in a more effective way, this paper proposed a novel feature extraction method for soft faults in non-linear analog circuits based on Local Mean Decomposition-Generalized Fractal Dimension (LMD-GFD) and Kernel Principal Component Analysis (KPCA). First, the fault signals were subject to LMD, the features of each component signal were extracted by GFD for the first time, and a high-dimensional feature space was formed. Then, KPCA was employed to reduce the dimensionality of the high-dimensional feature space, and feature extraction was performed again; at last, KPCA and Support Vector Machine (SVM) were adopted to diagnose the faults. The experimental results showed that the proposed LMD-GFD-KPCA method had effectively extracted the features of the soft faults in the non-linear analog circuits, and it achieved a high diagnosis rate.
\end{abstract}

Keywords: Fault Feature Extraction; Generalized Fractal Dimension (GFD); Kernel Principal Component Analysis (KPCA); Local Mean Decomposition (LMD); Nonlinear Analog Circuit

\section{INTRODUCTION}

At present, nonlinear analog circuits are widely used in electronic systems in aerospace, military, communication and other fields. Statistics show that the soft fault rate is more than $80 \%$, far more than the hard fault probability. In order to further enhance the reliability of these electronic products and equipment and effectively shorten the production time, higher requirements have been posed on the soft fault diagnosis technology of nonlinear analog circuits $[1,2]$. Because the soft faults of nonlinear analog circuits are complex and diverse, and the circuits also have the characteristics of non-linear response and tolerance of component parameters, in actual engineering practice it is difficult to effectively diagnose the faults or extract features of fault status using traditional methods, and the diagnosis efficiency of these methods is generally not satisfactory $[3,4]$.

The Volterra kernel analysis method and Wiener kernel analysis method proposed in recent years have further developed the practicability of feature extraction and fault diagnosis theories $[5,6]$, but these methods only have good effects in terms of soft fault diagnosis of weakly nonlinear analog circuits. When the nonlinear feature of the analog circuit is more obvious, the order that represents the system feature is higher, and the computational complexity would increase. At present, developing easy-to-calculate and highly accurate fault diagnosis methods that are suitable for the soft faults of non-linear analog circuits is a key research topic in the field [7, 8]. Fractal theory is an important branch of nonlinear discipline; it can effectively describe local fractal features in nonlinear processes. By applying fractal theory to the soft fault diagnosis of nonlinear analog circuits, the fractal dimension can be used to accurately describe the fault features of the circuits and effectively identify the soft fault status of the analog circuits. At present, the research of using the single-fractal dimension in fractal theory to describe the features of soft faults of analog circuits has received extensive attention. For example, Badoni et al. [9] used wavelet fractal method to diagnose the faults of the three-phase bridge rectifier circuit. Liu et al. [10] employed SVM and analog circuit fault diagnosis method with fractal features, and achieved good effects. Zhou et al. [11] adopted FRFT (fractional Fourier transform) fractal dimension to extract the fault features of analog circuits. These methods extracted circuit fault features from the perspective of single fractal and had achieved good results. However, single fractal can only analyze global features, and its performance is not good enough in case of describing local features of the signals. Multi-fractal is a collection of multiple measures, it can well reflect the signal features from both the global and the local scales. Lu et al. [12] applied EMD and generalized multifractal to fault signal feature extraction, and achieved accurate description of the local fractal features of fault signals. However, because the features extracted by GFD are usually of higher dimensions, inevitably there's redundant information in the extracted high-dimensional features; moreover, after fault signals were subject to EMD, as feature signals increased, redundant information would further increase. At the same time, since the EMD algorithm itself has the end effect, mode mixing effect, and negative frequency phenomenon, therefore, on the premise of accurately decomposing the fault signals, it is necessary to extract the high-dimensional features and perform dimensionality reduction, so as to realize the classification of different faults.

LMD is an improved method based on EMD, especially in terms of the processing effect of the highfrequency band of signals. It has a higher identification degree than EMD, and it can fully reflect the detailed features of the faults [13]. KPCA maps the input vector to a high-dimensional feature space through the selected nonlinear kernel function, so that the input vector has better separability in this space. Then, it performs PCA on the high-dimensional space data to obtain the non-linear principal components, thereby realizing the function of feature extraction [14].

This paper proposed a method for soft fault feature extraction of nonlinear analog circuits based on LMD-GFD and KPCA. First, the proposed method used LMD to decompose the fault signals, then, it calculated the generalized multifractal dimension of the effective component signals to obtain a high-dimensional feature space; at last, it reduced the dimensions of the high- 
dimensional feature space using KPCA to achieve the purpose of fault feature extraction.

\section{LMD}

LMD uses smoothing to generate local mean value function and local envelope function, it separates the pure frequency modulated (FM) signals and envelope signals from the complex multi-component signals and multiplies them to obtain a series of product functions (PF) with physically meaningful instantaneous frequency and the sum of a monotonic function $y_{k}(t)$. The decomposition steps are as follows:

1) Determine all local extreme points $n_{i}$ of original signal $x(t)$, and calculate the average value $m_{i}$ and estimated envelope value $a_{i}$ of all adjacent local extreme points:

$m_{i}=0.5\left(n_{i}+n_{i+1}\right)$

$a_{i}=0.5\left|n_{i}-n_{i+1}\right|$

2) Use broken lines to connect all adjacent average values $m_{i}$ and estimated envelope values $a_{i}$, and then use the moving average method to perform smoothing and obtain the local mean function $m_{11}(t)$ and envelope estimation function $a_{11}(t)$;

3) Separate the local mean function $m_{11}(t)$ from the original signal $x(t)$ to get $h_{11}(t)$, which is then divided by the envelope estimation function $a_{11}(t)$ to demodulate to get the FM signal $s_{11}(t)$.

$h_{11}(t)=x(t)-m_{11}(t)$

$s_{11}(t)=h_{11}(t) / a_{11}(t)$

4) Calculate the envelope estimation function $a_{12}(t)$ of $s_{11}(t)$, if $a_{12}(t)$ is not equal to 1 , then $s_{11}(t)$ is not a pure FM signal. Repeat the iteration for $\mathrm{n}$ times until $s_{1 n}(t)$ is a pure FM signal. In order to get good decomposition effect, the iteration termination condition can be set as follows:

$\lim _{n \rightarrow \infty}\left|a_{1 n}(t)-1\right| \leq \varepsilon$

Among $\varepsilon$ it is a very small variable.

5) Multiply the envelope estimation functions generated by the iterative process to obtain the envelope signal $a_{1}(t)$, and multiply it with the pure frequency modulation function $s_{1 n}(t)$ to obtain the product function $P F_{1}(t)$ of signal $x(t)$ :

$$
\begin{aligned}
& a_{1}(t)=a_{11}(t) a_{12}(t), \ldots, a_{1 n}(t)=\coprod_{j}^{n} a_{1 j}(t) \\
& P F_{1}(t)=a_{1}(t) s_{1 n}(t)
\end{aligned}
$$

$P F(t)$ is a single component FM AM signal, which corresponds to the highest frequency component in the original signal.

6) Separate $P F_{1}(t)$ from the original signal $x(t)$ to obtain signal $y_{1}(t)$, and repeat the above steps for $k$ times with $y_{1}(t)$ as the original data until $y_{k}(t)$ is a monotonic function.
7) The obtained signal $x(t)$ is equivalent to the sum of all PF components and $y_{k}(t)$ :

$$
x(t)=\sum_{i}^{k} P F_{i}(t)+y_{k}(t)
$$

\section{$3 \quad \mathrm{KPCA}$}

Kernel function aims to solve the non-nonlinear or linear inseparable problems in the measurement space. By searching for the suitable nonlinear mapping function $\Phi(X)$, it maps the sample set $X$ in the measurement space to a higher-dimensional space $F$, so as to classify the linear inseparable problems in space $F$.

Non-linear mapping function $\Phi: R^{m} \rightarrow F$ maps the input space $(k=1,2, \ldots, n)$ to the feature space $F: \Phi\left(x_{k}\right),(k$ $=1,2, \ldots, n)$. Suppose the mean value of $F: \Phi\left(x_{k}\right),(k=1$, $2, \ldots, n)$ has already been removed, then the covariance matrix in space $F$ is:

$C_{F}=\frac{1}{n} \sum_{i=1}^{n} \Phi\left(x_{i}\right) \Phi\left(x_{i}\right)^{T}$

Perform eigenvector analysis on matrix $C_{F}$. Suppose its eigenvalue is $\lambda$, eigenvector is $V, \lambda V=C_{F} V$, find the inner product of each sample and this formula, then we can get:

$\lambda\left[\Phi\left(x_{k}\right), V\right]=\left[\Phi\left(x_{k}\right), C_{F} V\right](k=1,2, \ldots, n)$

Then, the linear expression $\Phi\left(x_{k}\right)$ of eigenvector $V$ can be expressed as:

$V=\sum_{i=1}^{n} \alpha_{j} \Phi\left(x_{i}\right)$

where, $\alpha_{j}$ is the correlation coefficient.

By combining Eq. (9) to Eq. (11), we can get:

$$
\begin{aligned}
& \lambda \sum_{i=1}^{n} \alpha_{j}\left[\Phi\left(x_{k}\right), \Phi\left(x_{j}\right)\right]= \\
& =\frac{1}{n} \sum_{i=1}^{n} \alpha_{j}\left[\Phi\left(x_{k}\right), \sum_{i=1}^{n} \Phi\left(x_{i}\right)\right]\left[\Phi\left(x_{i}\right), \Phi\left(x_{j}\right)\right]
\end{aligned}
$$

Define a square matrix $\boldsymbol{K}$ with $n \times n$ :

$\boldsymbol{K}_{i j}=\left[\Phi\left(x_{i}\right), \Phi\left(x_{j}\right)\right]$

Then Eq. (12) can be simplified to $n \lambda \boldsymbol{K} \alpha=\boldsymbol{K}^{2} \alpha$, from which it can be seen that, performing linear PCA in feature space $F$ is equivalent to solving the eigenvalues and eigenvectors of Eq. (13). For the eigenvalues of matrix $\boldsymbol{K}$, there are $\lambda_{1} \geq \lambda_{2} \geq, \ldots, \geq \lambda_{n}$; and the corresponding eigenvectors are $\alpha^{1}, \alpha^{2}, \ldots, \alpha^{\mathrm{n}}$. In order to achieve the purpose of dimensionality reduction, the first $p(p \leq n)$ eigenvalues and eigenvectors could be retained. The matrix $K$ could be determined by selecting the kernel function.

To extract the principal component features, calculate the projection of the mapping data in feature space $F$ : 


$$
t_{k}=\left[V^{k}, \Phi(x)\right]=\sum_{j=1}^{n} \alpha_{j}^{k}\left[\Phi\left(x_{j}\right), \Phi(x)\right]
$$

At present, the commonly used kernel functions include the Gaussian radial basis kernel function, and the polynomial kernel function, etc. The number of principle components can be determined by the contribution of the principle components; the definition is:

$$
C\left(\lambda_{k}\right)=\frac{\lambda_{k}}{\sum_{j=1}^{n} \lambda_{j}} \times 100 \%
$$

where, $C\left(\lambda_{k}\right)$ is the contribution rate of the $k$-th principle component, it represents the percentage of the system information contained in the $k$-th principle component in all information.

In order to realize both the maximum original information retained and the reduced dimensionality of the feature space, it is required that the cumulative contribution rate $\mathrm{CPV}$ of the principle components $p$ must be greater than a certain limit value, namely:

$$
\mathrm{CPV}=\frac{\sum_{j=1}^{p} \lambda_{j}}{\sum_{j=1}^{n} \lambda_{j}} \geq C^{\prime}
$$

\section{EXPERIMENT AND SIMULATION}

The circuit of Sallen-key with filter is used to verify LMD-GFD-KPCA. Fig. 1 shows the circuit.

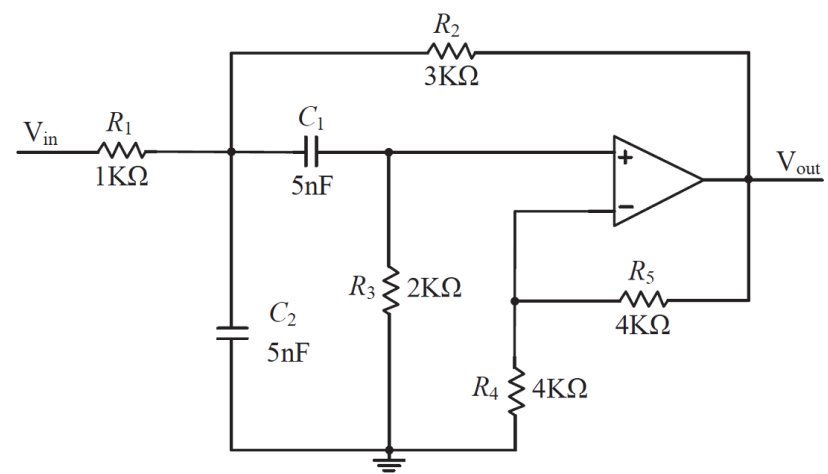

Figure 1 Sallen-key bandpass filter circuit

The excitation signal for this experiment was $\sin (800 \pi t)+\sin \sin (2000 \pi t)+\sin \sin (3200 \pi t)$, and the resistance capacitance in the circuit had a 5\% tolerance; that is, the component value varied within the tolerance range, which implied that this circuit was normal. The circuit was a bandpass filter with a center frequency of 25 $\mathrm{kHz}$ and a bandwidth of $50 \mathrm{kHz}$. In this test, $20 \%$ of the nominal value of the positive and negative drift was set as the fault state. (Too high positive and negative drift value will directly lead to structural failure, making the circuit unable to complete the basic work. So this is set to $20 \%$.) Only the signals of the output node $V$ out were collected and analyzed, and other nodes are not discussed here. A total of fifteen kinds of parameter-type fault combinations were configured. The failure of amplifier is affected by many factors, so it is not discussed here. The fault mode settings are shown in Tab. 1.

Table 1 Fault mode settings

\begin{tabular}{|c|c|c|c|}
\hline Fault Code & Fault State & Fault Code & Fault State \\
\hline 0 & Normal & 8 & $R_{4}-20 \%$ \\
\hline 1 & $R_{1}+20 \%$ & 9 & $R_{5}+20 \%$ \\
\hline 2 & $R_{1}-20 \%$ & 10 & $R_{5}-20 \%$ \\
\hline 3 & $R_{2}+20 \%$ & 11 & $C_{1}+20 \%$ \\
\hline 4 & $R_{2}-20 \%$ & 12 & $C_{1}-20 \%$ \\
\hline 5 & $R_{3}+20 \%$ & 13 & $C_{2}+20 \%$ \\
\hline 6 & $R_{3}-20 \%$ & 14 & $C_{2}-20 \%$ \\
\hline 7 & $R_{4}+20 \%$ & & \\
\hline
\end{tabular}

With the signals of faults $1,2,3$, and 4 as examples, the fault signals collected at node Vout were subject to LMD to obtain the PF component. Then, the fault signals and the time series of LMD of the fault signals were subject to generalized multifractal calculations, the four types of faults and the generalized multi-fractal dimension spectrum of the LMD of the fault signals are shown in Fig. 2 to Fig. 5.

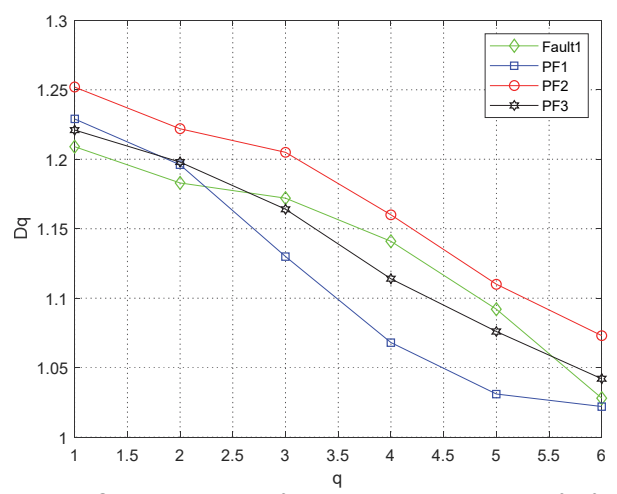

Figure 2 Generalized multi-fractal dimension spectrum for fault 1

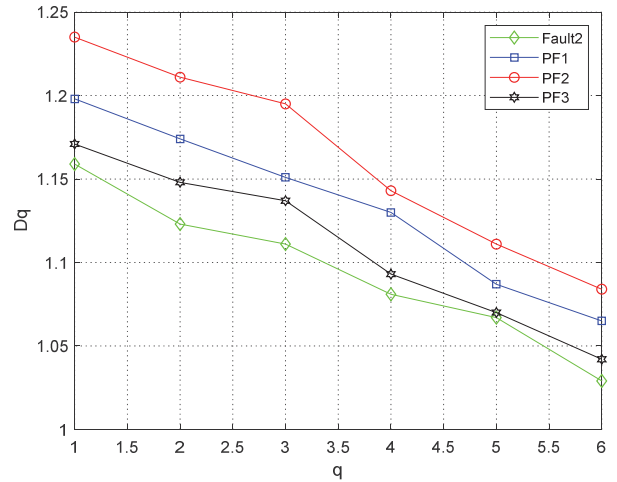

Figure 3 Generalized multi-fractal dimension spectrum for fault 2

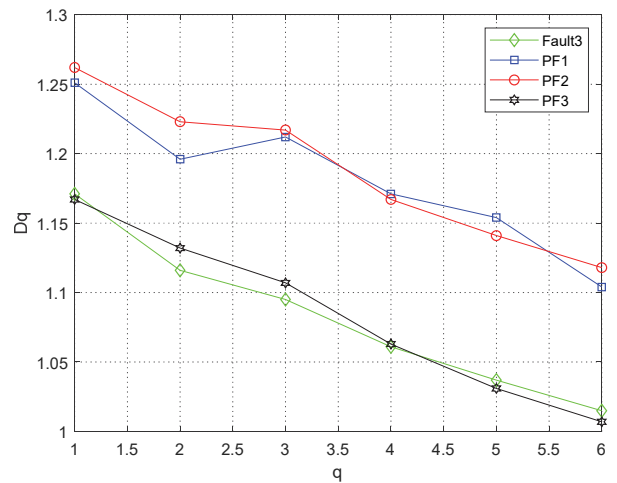

Figure 4 Generalized multi-fractal dimension spectrum for fault 3 


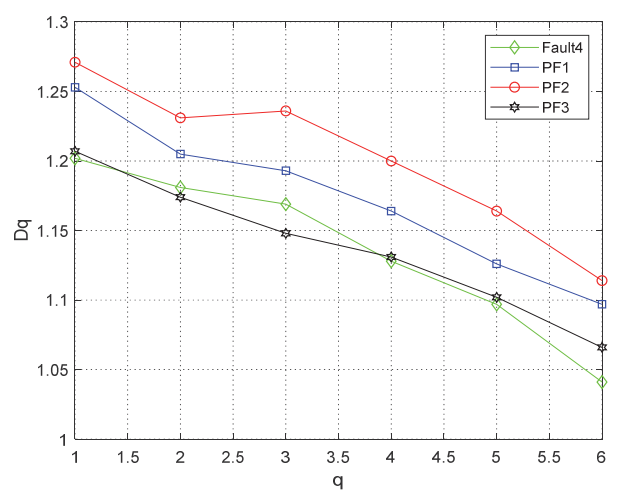

Figure 5 Generalized multi-fractal dimension spectrum for fault 4

The dimension sequence $D_{q}^{i}$ is the generalized fractal dimension of the $i$-th state. The PF component with high correlation coefficient with the original signal is selected. On the premise of dimension sequence $D_{q}^{x}$, the dimension correlation function of fault signal $\mathrm{x}$ is as follows:

$$
R(i, x)=1 / \sqrt{\frac{1}{n+1} \sum_{q=0}^{n}\left(D_{q}^{i}-D_{q}^{x}\right)^{2}} \quad q=0,1, \ldots, n
$$

The larger $R(i, x)$, the stronger the correlation between signal $x$ and $i$ states. The smaller $R(i, x)$, the weaker the correlation between signal $x$ and $i$ states.

The cross-correlation coefficient criterion is used here to filter $(q=3,4,5)$. Tab. 2 presents the average correlation coefficient between each PF component and the original signal. The correlation coefficients between the first three PF components (notated as PF1 - PF3) and the original signals are more greater than those of the remaining $\mathrm{PF}$ components and their original signals. From the fourth pf component, the correlation coefficient is far lower than the first three PF components, which contains too much false information and cannot accurately reflect the characteristics of the original signal. Therefore, PF1 - PF3 are selected as the principle PF component because they have a greater correlation with the original signals. Three generalized multi-fractal dimensions are picked from each of the three PF components. Hence, nine generalized multifractal dimensions for one fault signal are treated as its characteristic parameters (Tab. 3).

Table 2 Correlation coefficients between PFs and original signals

\begin{tabular}{|c|c|c|c|c|}
\hline Fault Code & PF1 & PF2 & PF3 & $\geq$ PF4 \\
\hline 1 & 60.97 & 32.26 & 28.38 & $<1$ \\
\hline 2 & 58.27 & 39.80 & 18.58 & $<1$ \\
\hline 3 & 107.42 & 10.14 & 9.63 & $<1$ \\
\hline 4 & 71.49 & 27.86 & 15.19 & $<1$ \\
\hline
\end{tabular}

Table 3 Characteristic parameters of generalized multi-fractal of fault signals

\begin{tabular}{|c|c|c|c|c|c|c|c|c|c|}
\hline Fault Code & $D_{2}^{P F 1}$ & $D_{3}^{P F 1}$ & $D_{4}^{P F 1}$ & $D_{2}^{P F 2}$ & $D_{3}^{P F 2}$ & $D_{4}^{P F 2}$ & $D_{2}^{P F 3}$ & $D_{3}^{P F 3}$ & $D_{4}^{P F 3}$ \\
\hline 0 & 1.147 & 1.122 & 1.016 & 1.137 & 1.096 & 1.084 & 1.167 & 1.131 & 1.124 \\
\hline 1 & 1.183 & 1.172 & 1.141 & 1.196 & 1.130 & 1.068 & 1.222 & 1.205 & 1.160 \\
\hline 2 & 1.123 & 1.111 & 1.081 & 1.174 & 1.151 & 1.130 & 1.211 & 1.195 & 1.143 \\
\hline 3 & 1.116 & 1.095 & 1.061 & 1.196 & 1.212 & 1.171 & 1.223 & 1.217 & 1.167 \\
\hline 4 & 1.181 & 1.169 & 1.128 & 1.205 & 1.193 & 1.164 & 1.231 & 1.236 & 1.200 \\
\hline 5 & 1.121 & 1.120 & 1.066 & 1.075 & 1.057 & 1.057 & 1.119 & 1.092 & 1.078 \\
\hline 6 & 1.126 & 1.107 & 1.034 & 1.113 & 1.104 & 1.046 & 1.132 & 1.099 & 1.069 \\
\hline 7 & 1.117 & 1.084 & 1.061 & 1.108 & 1.109 & 1.065 & 1.211 & 1.201 & 1.180 \\
\hline 8 & 1.127 & 1.118 & 1.107 & 1.119 & 1.114 & 1.103 & 1.203 & 1.203 & 1.175 \\
\hline 9 & 1.122 & 1.089 & 1.075 & 1.103 & 1.104 & 1.089 & 1.112 & 1.097 & 1.087 \\
\hline 10 & 1.124 & 1.114 & 1.061 & 1.102 & 1.091 & 1.020 & 1.158 & 1.087 & 1.049 \\
\hline 11 & 1.123 & 1.111 & 1.087 & 1.109 & 1.101 & 1.090 & 1.124 & 1.122 & 1.100 \\
\hline 12 & 1.102 & 1.068 & 1.008 & 1.098 & 1.088 & 1.065 & 1.075 & 1.075 & 1.057 \\
\hline 13 & 1.152 & 1.112 & 1.100 & 1.108 & 1.097 & 1.078 & 1.107 & 1.099 & 1.091 \\
\hline 14 & 1.074 & 1.049 & 1.047 & 1.102 & 1.102 & 1.050 & 1.111 & 1.103 & 1.084 \\
\hline 15 & 1.102 & 1.066 & 1.049 & 1.088 & 1.075 & 1.047 & 1.094 & 1.087 & 1.084 \\
\hline
\end{tabular}

GFD was used to extract the features of different status of the soft faults in nonlinear analog circuits, and a 9dimensional feature space was generated. Since in highdimensional feature spaces, there is certain redundancy between the features, the dimensionality needs to be reduced.

At present, the kernel function forms to perform KPCA on the feature set of the original status are mainly divided into the following categories:

Gaussian kernel function, also known as radial basis kernel function:

$$
K\left(x_{i}, x_{j}\right)=\exp \left(-\frac{\left\|x_{i}-x_{j}\right\|^{2}}{2 \sigma^{2}}\right)
$$

Exponential kernel function:

$$
K(x, y)=\exp \left(-\frac{\|x-y\|}{2 \sigma^{2}}\right)
$$

Polynomial kernel function:

$$
K(x, \mathrm{y})=\left(\langle x \cdot \mathrm{y}\rangle+d_{i}\right)^{d_{2}}
$$

Kernel function of perceptron:

$$
K(x, y)=\tan h\left(\beta_{0}\langle x \cdot y\rangle+\beta_{1}\right)
$$

Among them, the nuclear parameters can be determined by experience according to different situations. Gaussian radial basis kernel function is superior to other kernel functions in prediction speed and accuracy. Gaussian radial basis kernel function is used in most problems. This paper chose to use the Gaussian radial basis 
kernel function Eq. (18) to perform KPCA on the feature set of the original status, and to realize dimensionality reduction of high-dimensional feature spaces and fault classification.

Contribution rate of the principle components of faults 1 - 4 was calculated by KPCA algorithm and the results are shown in Tab. 4. The limit value of the cumulative contribution rate was set to $98 \%$. It can be concluded that the first three principal components can represent all status information. In this way, the dimensionality reduction from 9 dimensions to 3 dimensions could be realized.

Table 4 Cumulative contribution rate

\begin{tabular}{|c|c|c|c|}
\hline Cumulative contribution rate & KPCA1 & KPCA2 & KPCA3 \\
\hline Fault 1/\% & 95.74 & 97.82 & 98.76 \\
\hline Fault 2/\% & 95.24 & 97.06 & 98.23 \\
\hline Fault 3/\% & 97.34 & 99.76 & 100 \\
\hline Fault 4/\% & 97.62 & 99.88 & 100 \\
\hline
\end{tabular}

The feature extraction results based on the LMD-GFDKPCA method are shown in Fig. 6. According to the figure, the separability of each fault category was good.

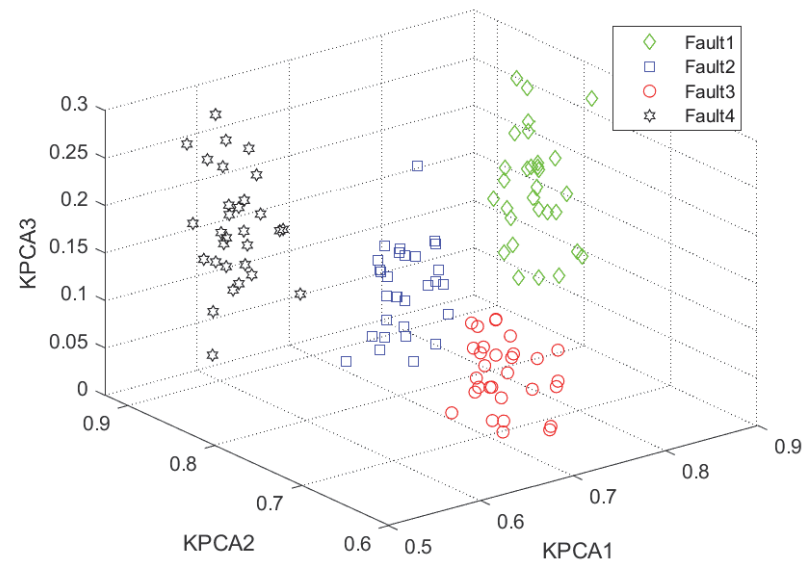

Figure 6 Feature extraction based on LMD-GFD-KPCA

To further verify the effect of the proposed feature extraction method, SVM was used to model and classify the extracted features. Then, a comparison study was conducted on three methods, namely the proposed LMDGFD-KPCA method, the KPLS-WNN method (a WNN analog circuit soft fault diagnosis method based on KPLS feature extraction proposed in Cong et al. [15]), and the EMD-MFD method (a generalized multifractal fault diagnosis method based on empirical mode decomposition proposed in Lu et al. [12]), and the diagnosis results of the three methods are shown in Tab. 5 .

Table 5 Fault diagnosis results of three methods

\begin{tabular}{|l|c|c|c|}
\hline & $\begin{array}{c}\text { KPLS- } \\
\text { WNN }\end{array}$ & $\begin{array}{c}\text { EMD- } \\
\text { MFD }\end{array}$ & $\begin{array}{c}\text { LMD-GFD- } \\
\text { KPCA }\end{array}$ \\
\hline Average detection rate $/ \%$ & 96.7 & 96.9 & 97.5 \\
\hline
\end{tabular}

According to Tab. 5, it can be concluded that the proposed LMD-GFD-KPCA method outperformed the other two methods in diagnosis accuracy.

\section{CONCLUSIONS}

When using the generalized multifractal dimension to extract features of fault signals, the obtained features are often of high dimensions and there's redundant information in the features. Aiming at this problem, this paper proposed a new fault feature extraction method based on LMD-GFD and KPCA. First, the fault signals were subject to LMD to obtain several PF components, the correlation between the decomposed signals and the original signals was calculated by the related functions, then, the proper PF component signal was selected, and the GFD was calculated to obtain a high-dimensional feature set; after that, KPCA was used to reduce the dimensionality, and a fault signal feature set after dimensionality reduction was obtained; at last, SVM was adopted for classification and diagnosis, and the experimental results proved that the proposed fault feature extraction method based on LMD-GFD and KPCA showed good performance.

\section{Acknowledgments}

The authors wish to thank Scientific Research Project of Science and Engineering Talents Program of Harbin University of Science and Technology (No. LGYC2018JC047) and Heilongjiang Provincial Leading Talent Echelon Reserve Leader Funding for their support.

\section{REFERENCES}

[1] Sobanski, P. \& Kaminski, M. (2019). Application of artificial neural networks for transistor open-circuit fault diagnosis in three-phase rectifiers. IET Power Electronics, 12(9), 2189-2200. https://doi.org/10.1049/iet-pel.2018.5330

[2] Rudsari, F. N., Razi-Kazemi, A. A., \& Shoorehdeli, M. A. (2019). Fault Analysis of High-Voltage Circuit Breakers Based on Coil Current and Contact Travel Waveforms through Modified SVM Classifier. IEEE Transactions on Power Delivery, 34(4), 1608-1618. https://doi.org/10.1109/TPWRD.2019.2915110

[3] Gan, X. S., Qu, H., Meng, X. W., Wang, C. L., \& Zhu, J. (2019). Research on ELM soft fault diagnosis of analog circuit based on KSLPP feature extraction. IEEE Access, 7: 92517-92527. https://doi.org/10.1109/ACCESS.2019.2923242

[4] Sanatescu, D. R., Ene, L. V., Ionescu, A., Orosanu, A., \& Iordache, M. (2019). A new approach for nonlinear analog circuits analysis. UPB Scientific Bulletin, Series C: Electrical Engineering and Computer Science, 81(3), 197208.

[5] Borys, A. (2016). On Definition of Operator o for Weakly Nonlinear Circuits. International Journal of Electronics and Telecommunications, 62(3), 253-259. https://doi.org/10.1515/eletel-2016-0034

[6] Lin, H. J., Zhang, L. Y., Ren, D. Y., Kang, H., \& Gu, G. (2009). Fault diagnosis in nonlinear analog circuit based on Wiener kernel and BP neural network. Chinese Journal of Scientific Instrument, 30(9), 1946-1949.

[7] Lin, Q. B., Chen, S. C., \& Lin, C. (2019). Parametric fault diagnosis based on fuzzy cerebellar model neural networks. IEEE Transactions on Industrial Electronics, 66(10), 81048115. https://doi.org/10.1109/TIE.2018.2884195

[8] Bayma, R., Teixeira, R., Lopes, D., \& Tavares, C. (2019). A differential approach for obtaining diagonal volterra kernels. IEEE Transactions on Circuits and Systems II: Express Briefs, 66(8), 1481-1485. https://doi.org/10.1109/TCSII.2018.2889272

[9] Badoni, M., Singh, A., \& Singh, B. (2016). Comparative Performance of Wiener Filter and Adaptive Least Mean Square based Control for Power Quality Improvement. IEEE Transactions on Industrial Electronics, 63(5), 3028-3037. https://doi.org/10.1109/TIE.2016.2515558 
[10] Liu, M. R., He, Y. G., Fang, G. F., Yin, X., Qi, S. Z. (2009). Application for Fault Diagnosis of Analog Circuits Based on GA. Wavelet and NN. Journal of Hunan University (Natural Sciences), 36(3), 40-44.

[11] Zhou, S. L., Liao, J., \& Shi, J. J. (2014). New Method to Extract Analog Circuit Fault Features Based on FrFT-FD and KPCA. Journal of Vibration, Measurement and Diagnosis, 34(2), 337-344.

[12] Lu, X. M., Zhao, H., Wu, Q. (2016). Generalized Multifractal Method for Soft Fault Feature Extraction of Nonlinear Analog Circuits Based on EMD. Revista de la Facultad de Ingeniería, 31(7), 192-203.

[13] Li, G. L., Huo, M. X., Gao, X. Z., Miao, K., Ren, X. J., Qi, W. Y., \& Zhu, J. P. (2018). Fault Diagnosis Method of Circuit Breaker based on LMD and Time Spectrogram Fractal Dimension. Instrumentation Analysis Monitoring, 4, $1-5$.

[14] Wei, X., Li, B. W., \& Wu, Y. M. (2019). Vibration Feature Extraction Based on Generalized Fractal Dimension and Kernel Principal Component Analysis. Journal of Vibration, Measurement and Diagnosis, 39(1), 32-38.

[15] Cong, W., Jing, B., \& Yu, H. K. (2014). WNN soft fault diagnosis of analog circuit based on KPLS feature extraction. Journal of Central South University (Science and Technology), 45(6), 1841-1846.

\section{Contact information:}

\section{Xinmiao LU}

(Corresponding author)

School of Measurement-Control Technology and Communications Engineering,

Harbin University of Science and Technology,

Harbin 150080, China

E-mail: Ivxinmia00611@126.com

\section{Jiaxu WANG}

School of Measurement-Control Technology and Communications Engineering, Harbin University of Science and Technology,

Harbin 150080, China

E-mail:347690745@qq.com

\section{Qiong WU}

Heilongjiang Network Space Research Center,

Harbin 150090, China

E-mail: aa24928409@163.com

\section{Yuhan WEI}

School of Measurement-Control Technology and Communications Engineering, Harbin University of Science and Technology,

Harbin 150080, China

E-mail: 1360057108@qq.com

\section{Yanwen SU}

School of Measurement-Control Technology and Communications Engineering, Harbin University of Science and Technology,

Harbin 150080, China

E-mail: 1807673013@qq.com 